국내 야생화들로부터 국내 미기록 효모, Rhodosporidium fluviale, Rhodosporidium paludigenum 과 Candida sp. 80$\mathrm{J}-3$, Kluyveromyces thermotolerans의 특성

현세희 ${ }^{1} \cdot$ 이힝범 ${ }^{2} \cdot$ 이종수 ${ }^{*}$

${ }^{1}$ 배재대학교 바이오의생명공학과, ${ }^{2}$ 전남대학교 응용생물공학부

\title{
Characteristics of Unrecorded Yeasts, Rhodosporidium fluviale, Rhodosporidium paludigenum, Candida sp. 80-J- 3 and Kluyveromyces thermotolerans Isolated from Wild Flowers in Korea
}

\author{
Se-Hee Hyun', Hyang Burm Lee ${ }^{2}$ and Jong-Soo Lee ${ }^{1 *}$ \\ 'Department of Biomedicinal Science and Biotechnology, Paichai University, Daejeon 302-735, Korea \\ 2Divison of Applied Bioscience \& Biotechnology, Chonnam National University, Gwangju 500-757, Korea
}

\begin{abstract}
Rhodosporidium fluviale 201-C-1 and Kluyveromyces thermotolerans 150-JE-2(1) were isolated from wild flowers in Geumsan-gun, Chungcheongnam-do and Wando-gun, Jeollanam-do, respectively. Rhodosporidium paludigenum 86-J-1 and Candida sp. 80-J-3 were also isolated from flowers in Jeju island, Korea. Characteristics for these yeasts were not reported before. Therefore, morphological and physiological characteristics for these yeasts were investigated in this study. $R$. fluviale 201-C-1 was halophilic asporogenous yeast and formed pseudomycelium. It also grew at $30^{\circ} \mathrm{C}$ and $\mathrm{pH}$ 4.0-6.0. K. thermotolerans $150-\mathrm{JE}-2(1)$ was sugar-tolerant and halotolerant asporogenous yeast. It formed pseudomycelium and showed positive urease activity. Candida sp. 80-J-3 and R. paludigenum 86-J-1 were asporogenous yeasts, and grew well in yeast extract-peptone dextrose(YPD) medium, potato-dextrose medium as well as vitamin-free medium and YPD medium containing $5 \% \mathrm{NaCl}$.
\end{abstract}

KEYWORDS : Candida sp. 80-J-3, Kluyveromyces thermotolerans, New records yeasts, Rhodosporidium fluviale, Rhodosporidium paludegenum, Wild flowers

지금까지 꽃과 과일 등의 자연환경과 곡류 등의 농산물 등으로부터 유용효모의 분리 및 이용 연구와 국내 미기록

Kor. J. Mycol. 2013 June, 41(3): 181-184

http://dx.doi.org/10.4489/KJM.2013.41.3.181

pISSN 0253-651X

(C) The Korean Society of Mycology

*Corresponding author

E-mail: biotech8@pcu.ac.kr

Received July 29, 2013

Revised September 23, 2013

Accepted September 23, 2013

@This is an Open Access article distributed under the terms of the Creative Commons Attribution Non-Commercial License (http:// creativecommons.org/licenses/by-nc/3.0/) which permits unrestricted non-commercial use, distribution, and reproduction in any medium, provided the original work is properly cited.
효모들의 탐색 연구는 거의 실시되지 않았다. 최근 필자 등 은 환경부 국립 생물자원관의 지원으로 국내 논, 밭, 하천 및 야산 등지에 서식하고 있는 야생화들로부터 효모들을 분리, 동정하여 이들의 다양성 (Min et al., 2012; Kang et al., 2012; Min et al., 2013a)과 수십 종의 국내 미기록 효모 들을 선발하여 이들의 특성을 보고(Hyun et al., 2013a) 하 였고 경기도와 제주도 등지의 야생화들에서 효모들을 분 리, 동정한 후 항통풍성 Xanthin 산화효소 저해물질 생산 효모를 선발하였다(Hyun et al., 2013b).

본 연구에서는 충남 금산군과 전남 완도군 및 제주도에 서 서식하는 야생화들로부터 분리한 효모 균주들 중 지금 까지 국내에 보고되지 않은 Rhodosporidium fluviale 201C-1과 Rhodosporidium paludigenum 86-J-1, Candida sp. 80-J-3과 Kluyveromyces thermotolerans 150-JE-2(1) 효모들 
을 각각 선발하여(Table 1) 이들의 형태학적 및 배양, 생리 학적 특성들을 조사하였다.

충남 금산군과 전남 완도군 일대에서 2012년 개화한 꽃 (화분)들로부터 전보(Min et al., 2012)와 같이 효모들만을 선택적으로 분리한 후, $26 \mathrm{~S} \mathrm{rDNA}$ 의 $\mathrm{D} 1 / \mathrm{D} 2$ 부위 혹은 ITS1/5.8S rDNA/ITS2 부위의 염기서열 상동성 비교법을 이용하여 동정한 균들을 대상으로(Min et al., 2013a) RISS 와 PubMed 및 배재대학교 도서관 등의 균학 관련 논문 자 료들을 이용하여 국내에 아직까지 보고되지 않은 균주들을 선발하여 최종 국내 미기록 효모들로 확정하였다.

선정된 미기록 효모들에 대하여 광학현미경(DM IRE2, LEICA, Germany)과 전보와 이등(Kang et al., 2012; Lee et al., 1996)의 일반 미생물과 효모의 분리, 동정 실험방법 등
을 이용하여 형태학적 특징과 배양 및 주요 생리적 특성 등 을 조사하였다.

\section{국내 미기록 효모들의 특성}

충남 금산군 명곡리 개삼터 부근의 뚝갈(Patrinia villosa) 꽃에서 분리, 동정한 Rhodosporidium fluviale 201-C-1은 무포자 효모로 $20 \% \mathrm{NaCl}$ 을 함유한 YPD배지에서 생육하 는 내염성을 보였으며, urease 활성을 갖고 있었다(Table 2, Fig. 1). 또한 전남 완도군 대문리 원추리(Hemerocallis fulva) 꽃에서 분리한 Kluyveromyces thermotolerans 150-JE-2(1) 는 의균사를 형성하는 무포자 효모로 $50 \%$ 포도당과 $5 \%$ $\mathrm{NaCl}$ 을 각각 함유한 $\mathrm{YPD}$ 배지에서 생육이 양호한 내당성,

Table 1. Newly reporting yeasts from wild flowers of Geumsan-gun, Wando-gun and Jeju island, Korea

\begin{tabular}{|c|c|c|c|c|c|}
\hline Collected site & Putative species & Isolated No. & $\begin{array}{c}\text { Related Genbank } \\
\text { sequence }\end{array}$ & Identity (\%) & $\begin{array}{l}\text { Amplified } \\
\text { regions }\end{array}$ \\
\hline $\begin{array}{l}\text { Geumsan-gun, } \\
\text { Chungcheongnam-do }\end{array}$ & Rhodosporidium fluviale & 201-C-1 & FJ515184.1 & 598/607 (99) & $26 \mathrm{~S}$ rDNA \\
\hline $\begin{array}{l}\text { Wando-gun, } \\
\text { Jeollanam-do }\end{array}$ & Kluyveromyces thermotolerans & 150-JE-2(1) & DQ655683.1 & $568 / 572(99)$ & $26 \mathrm{~S}$ rDNA \\
\hline \multirow{2}{*}{$\begin{array}{l}\text { Songak Mt. } \\
\text { Jeju Island }\end{array}$} & Candida sp. & $80-\mathrm{J}-3$ & DQ655679.1 & $588 / 592(99)$ & $26 \mathrm{~S}$ rDNA \\
\hline & Rhodosporidium paludigenum & $86-J-1$ & HQ670676.1 & 605/607 (99) & $26 \mathrm{~S}$ rDNA \\
\hline
\end{tabular}

${ }^{\star}$ Newly reporting yeasts in this study were screened among the seventy one yeast strains isolated in Geumsan-gun and Wando-gun, Korea by Min et al. (2013b) and also thirty two yeast strains isolated in Jeju island by Hyun et al. (2013b).

Table 2. Morphological and cultural characteristics of the newly reporting yeasts, Rhodosporidium fluviale, Kluyveromyces thermotolerans, Candida sp. and Rhodosporidium paludigenum

\begin{tabular}{|c|c|c|c|c|}
\hline & $\begin{array}{l}\text { Rhodosporidium } \\
\text { fluviale 201-C-1 }\end{array}$ & $\begin{array}{l}\text { Kluyveromyces thermo- } \\
\text { tolerans } 150-J E-2(1)\end{array}$ & Candida sp. 80-J-3 & $\begin{array}{c}\text { Rhodosporidium } \\
\text { paludigenum 86-J-1 }\end{array}$ \\
\hline \multicolumn{5}{|l|}{ Morphological characteristics } \\
\hline Shape & $\mathrm{O}^{1)}$ & $\mathrm{O}$ & $\mathrm{O}$ & $\mathrm{E}^{2)}$ \\
\hline Vegetalle reproduction & $\mathrm{B}^{3)}$ & B & $\mathrm{B}$ & $\mathrm{B}$ \\
\hline Size $(\mu \mathrm{m})$ & $(4.0-6.0) \times(6.0-8.5)$ & $(4.0-5.5) \times(5.0-5.5)$ & $(10.3-10.9) \times(12.1-12.7)$ & $(13.5-14.1) \times(22.1-22.7)$ \\
\hline Ascospore & - & - & - & - \\
\hline Pseudomycelium & formed & formed & formed & - \\
\hline \multicolumn{5}{|c|}{ Cultural and physiological characteristics } \\
\hline Growth on YM & +++ & +++ & +++ & ++ \\
\hline Growth on YPD & +++ & + & +++ & +++ \\
\hline Growth on PD & ++ & ++ & +++ & ++ \\
\hline Color on YPD & $\mathrm{P}^{4)}$ & $C^{5)}$ & $\mathrm{W}^{6}$ & $\mathrm{C}$ \\
\hline Growth on Vitamin-free medium & $+^{7)}$ & + & ++ & ++ \\
\hline Growth in 50\% Glucose-YPD & - & ++ & + & + \\
\hline Growth in $5 \% \mathrm{NaCl}-\mathrm{YPD}$ & + & +++ & +++ & ++ \\
\hline Growth in $20 \% \mathrm{NaCl}-\mathrm{YPD}$ & + & - & - & - \\
\hline Growth in temp./pH range & $30^{\circ} \mathrm{C} / \mathrm{pH} \mathrm{4-6}$ & $30^{\circ} \mathrm{C} / \mathrm{pH} 4-6$ & $25-30^{\circ} \mathrm{C} / \mathrm{pH} 4-7$ & $25-30^{\circ} \mathrm{C} / \mathrm{pH} 7-8$ \\
\hline Urease activity & + & + & - & + \\
\hline
\end{tabular}




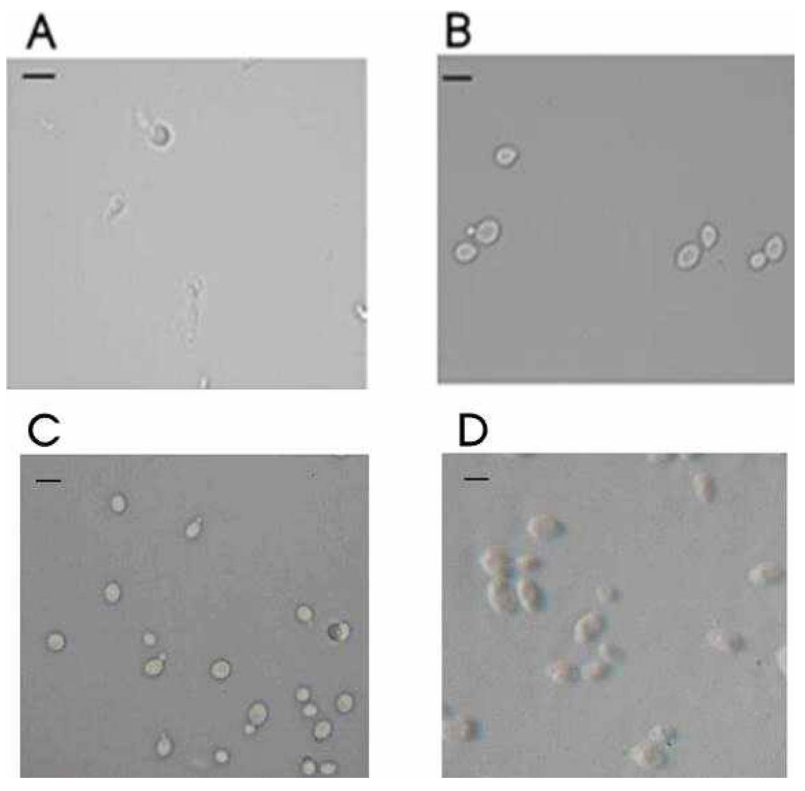

Fig. 1. Microscopic features of unrecorded yeasts, Rhodosporidium fluviale 201-C-1(A), Kluyveromyces thermotolerans 150JE-2(1)(B), Candida sp. 80-J-3(C) and Rhodosporidium paludigenum $86-\mathrm{J}-1(\mathrm{D})$. Scale bar is $5 \mu \mathrm{m}$.

호염성 효모이면서 $30^{\circ} \mathrm{C}$ 에서도 잘 생육하였고 urease 활성 을 갖고 있었다.

한편, 제주도 송악산 기슭의 자주색달개비 (Setcreasea purpurea Boom) 꽃으로부터 분리된 Candida sp. 80-J-3과 물 봉선(Impatiens textori Miquel) 꽃으로부터 분리된 Rhodosporidium paludigenum 86-J-1 두 효모들은 모두 출아에 의 한 무성생식을 하는, 무포자 효모들로서 각각 구형과 타원 형이었다. 또한 두 균주 모두 Yeast extract-peptone-dextrose 배지와 Potato-dextrose 배지 그리고 vitamin-free 배지에서 잘 생육하였다. 특히 이들은 $5 \% \mathrm{NaCl}$ 을 함유한 $\mathrm{YPD}$ 배지 에서 잘 생육하는, 내염성 효모들이었다. 또한, Candida sp. 80-J-3은 의균사를 형성하였고 $\mathrm{pH}$ 4.0 7.0에서 생육하였으 며 urease 활성은 음성이었다. 그러나 Rodosporidium paludigenum 은 의균사를 형성하지 않았고 $\mathrm{pH} 7.0 \sim 8.0$ 에서 잘 생육하였으며 urease 활성은 양성을 나타내었다.

이들 국내 미기록 효모들에 대한 국외 주요 연구로 Rhodosporidium fluviale 201-C-1에 관한 연구는 거의 실시되지 않았고 Ponsone등(2011)은 Kluyveromyces thermotolerans 가 Aspergillus carbonarius와 Aspergillus niger의 생육을 억 제시키는 Mycotoxin의 일종인 ochratoxin A의 축적을 억 제하였음을 보고하였고, Christen과 Sauer(2011)은 Kluyveromyces thermotolerans의 호기성 포도당대사에 관련된 대사공학 연구를 실시하여 보고하였다.

또한 Nguyen등(2007)이 처음으로 암컷 뱀잠자리 표면으 로부터 Candida sp. 80-J-3을 분리, 동정하여 일부 형태적, 생리적 특성을 보고하였고, Rodosporidium paludigenum에 관한 연구도 이들이 주로 과일 등의 수확 후 질병방제에 대
한 응용성이 연구, 보고되었을 뿐이다(Wang et al., 2011; Wang et al., 2010).

\section{적 요}

충남 금산군과 전남 완도군, 제주도 송악산 등지에서 서 식하고 있는 야생화들로부터 Rhodosporidium fluviale 201C-1과 Kluyveromyces thermotolerans 150-JE-2(1), Rodosporidium paludigenum과 Candida sp. 80-J-3등의 국내 미 기록 효모들을 분리, 선발하여 이들의 미생물학적 특성들 을 조사하였다. Rhodosporidium fluviale 201-C-1은 내염성 의 무포자 효모로 의균사를 형성하였고 urease 활성을 갖고 있었다. Kluyveromyces thermotolerans 150-JE-2(1)는 내당 성과 내염성을 가진 무포자효모로서 $30^{\circ} \mathrm{C}, \mathrm{pH} 4.0 \sim 6.0$ 에서 잘 생육하였다. Candida sp. 80-J-3과 Rhodosporidium paludigenum 86-J-1 균주들은 모두 무성생식을 하는 무포자 효 모들이었고 YPD 배지, Vitamin-free 배지와 $5 \% \mathrm{NaCl}$ 을 함 유한 YPD 배지 등에서 잘 생육하였다. 또한 Candida sp. 80-J-3 균주의 urease 활성은 음성이었으나 Rhodosporidium paludigenum 86-J-1 균주는 urease 활성이 양성이면서 $\mathrm{pH}$ 7.0 8.0에서 잘 생육하였다.

\section{감사의 글}

본 논문은 환경부 재원으로 국립 생물자원관의 지원을 받아 수행된 연구(NIBR No 2013-02-001) 결과의 일부로 연구비 지원에 감사드립니다.

\section{참고문헌}

Christen, S. and Sauer, U. 2011. Intracellular characterization of aerobic glucose metabolism in seven yeast species by ${ }^{13} \mathrm{C}$ flux analysis and metabolomics. FEMS. Yeast Res. 11:263-272.

Hyun, S. H., Lee, H. B., Kim, C. M., Kim, H. K. and Lee, J. S. 2013a. New records of yeasts from wild flowers in coast near areas and inland areas, Korea. Kor. J. Mycol. 41:74-80. (in Korean).

Hyun, S. H., Mun, H. Y., Lee, H. B., Kim, H. K. and Lee, J. S. 2013b. Isolation of yeasts from wild flowers in Gyonggi-do and Jeju island of Korea and production of anti-gout xanthin oxidase inhibitor. Korean J. Microbiol. Biotechnol. 41(3). (Submitted, in Korean).

Kang, M. G., Hyun, S. H., Ryu, J. J., Min, J. H., Kim, H. K. and Lee, J. S. 2012. Note on newly isolated yeasts from wild flowers in Daejeon city, Korea. Kor. J. Mycol. 40:174-176. (in Korean).

Lee, J. S., Choi, Y. J., Kwon, S. J., Yoo, J. Y. and Chung, D. H. 1996. Screening and characterization of osmotolerant and gas-producing yeasts from traditional Doenjang and Kochujang. Food Biotechnol. 5:54-58.

Min, J. H., Hyun, S. H., Kang, M. G., Lee, H. B. Kim, C. M., Kim, H. K. and Lee, J. S. 2012. Isolation and identification of yeasts 
from wild flowers of Daejeon city and Chungcheongnam-do in Korea. Kor. J. Mycol. 40:141-144. (in Korean).

Min, J. H., Ryu, J. J., Kim, H. K. and Lee, J. S. 2013a. Isolation and identification of yeasts from wild flowers in Gyejoksan, Oseosan and Beakamsan of Korea. Kor. J. Mycol. 41:47-51. (in Korean)

Min, J. H., Lee, H. B., Lee, J. S. and Kim, H. K. 2013b. Identification of yeast isolated from wild flowers collected in coast areas of Korea based on the 26S rDNA sequences. Kor. J. Mycol. 41. (Submitted).

Nguyen N. H., S. O. Suh, and M. Blackwell. 2007. Five novel Candida species in inisect-associated yeast clades isolated from Neuroptera and other insects. Mycologia 99:842-858.
Ponsone, M. L., Chiotta, M. L., Combina, M., Dalcero, A. and Chulze, S. 2011. Biocontrol as a strategy to reduce the impact of ochratoxin A and Aspergillus section Nigri in grapes. Int. J. Food. Microbiol. 151:70-77.

Wang P., X. Liu, Y. Wang, H. Ruan, and X. Zheng. 2011. Statistical media optimization for the biomass production of postharvest biocontrol yeast. Prep. Biochem. Biotechnol. 41:382-397.

Wang Y., P. Wang, J. Xia, T. Yu, B. Lou, J. Wang, and X. D. Zheng. 2010. Effect of water activity on stress tolerance and biocontrol activity in antagonistic yeast Rhodosporidium paludigenum. Intl. J. Food Microbiol. 143:103-108. 